\title{
Air Flow Simulation and Optimization for Negative Pressure Safflower Harvesting Device
}

\author{
Ge Yun, Zhang Lixin*, Han Dandan, Zeng Haifeng and Zhang Xiang \\ College of Mechanical and Electrical Engineering, Shi Hezi University, Xinjiang, China
}

\begin{abstract}
The safflower collection device is an important service part of safflower harvesting device, the number of the air outlets and external structure of air collecting hood are the main factors influencing the effect of the flower's petals harvesting and collection. However, the design of pneumatic safflower harvest device is based on experience, resulting in long the design cycle, thus increasing the cost of design. Combined with the advantages of computational fluid dynamics software FLUENT, and the internal flow field of safflower closed style cover flowers as the research object, this paper designed gas collecting hood structures with different number of outlets through the analysis of the airflow distribution and motion law. The result of numerical simulation of gas-collecting hood with trapezoidal cone shows higher air flow speed with no low velocity zone, and the distribution of flow field of safflower collected cover with trapezoid cone type is better than that of parabolic type structure. Based on result of simulation, improved the design of the external shape of flowers cover and designed trapezoid cone type to improve the internal airflow state, thus could achieve the goal of improving efficiency of picking and collecting and reduce energy consumption.
\end{abstract}

Keywords: Fluid analysis, negative pressure, safflower, structure optimization.

\section{INTRODUCTION}

Safflowers have very high application value and have been greatly promoted in medicine and food industry, and also has great development potential [1-2]. With the gradual increase of the market demand for safflower, the traditional manual picking can not meet the production requirements [35]. And due to the shorter flowering period, the wilting safflower petals increase the difficulty of picking so there is the monumental waste.The safflower is light weight, small size, which are more suitable for a pneumatic mechanical harvest, so pneumatic could solve the problem of lack of labour force in harvest season. According to statistics, the planting area of safflower is more than 5 ten thousand ha in China in 2014, and producing $90 \%$ of the world's total output. However, safflower picked only rely on manual completely at present. This paper adopts the combination of the way that the positive pressure air flow to separates safflower and the positive pressure air flow to collecting and conveying safflower. This way can effectively improve the recovery efficiency and reduce the recovery cost.

However, the design of pneumatic safflower harvest device is based on experience, resulting the problems that eddy current and low utilization efficiency in the internal flow field of picking, collecting, conveying device. While based on the analysis of the theory of fluid mechanics, experimental analysis, and based on the computational fluid dynamics numerical simulation analysis of CFD (Comptational Fluid Dynamics) technology with the advantages of low cost, short period calculated and

*Address correspondence to this author at the College of Mechanical and Electrical Engineering, Shi Hezi University, Xinjiang, China;

Tel: +18999330088; E-mails: gy_shz@163.com visualization of simulation results, which makes up greatly the insufficiency of theoretical analysis and experimental analysis.

In recent years, the CFD technology is widely used in the process of mechanical design, such as applying computational fluid dynamics technology to analyze flow field simulation of the dust collecting system of vacuum sweeper and optimize the structure, using software Fluent to calculate the suction forces action on seeds in the gas field nearby vacuum nozzle [6-9]. Seed motion differential equation was derived and using central difference method and then the typical suction trajectory was received which could be described, using FLUENT software to simulate and experimental-ly study on the sucking nozzle air field, which affected sucking effect of air-suction seeder and using CFD to analyze airflow field distribution law of greenhouses and factors that influence picking robot vacuum negative pressure changes [10-12]. But there is rarely reported on pneumatic study of this small and lightweight materials like safflower which are separated from fruit ball by the air flow.

Combined with the biological characteristics of safflower, negative pressure collecting device collects and transports safflower petals to the storage device timely and effectively, therefore it is very important to research on the rules of the internal air flow in collection device for collecting safflowers effectively.

Therefore, this paper takes the safflower negative pressure collecting device as the research object, and makes the numerical simulation of the internal flow field of safflower pneumatic collection device by Fluent software and analyzes local, then establishes energy loss equation, finally optimizes and designs the structure parameters of safflower negative pressure collecting device 


\section{PRINCIPLE OF SAFFLOWER PNEUMATIC HARVESTER}

The principle of pneumatic safflower harvester is shown in Fig. (1). Positive pressure pneumatic harvesting device is placed on both sides of the safflower, there is the positive pressure airflow when the fan rotation, and safflower petals are separated from fruit ball under the action of wind which is form the outlets on both sides of the plants, then the separation of the petals fall down in the role of gravity or suspend in the air under the blowing airflow, finally under the action of negative pressure suction flow into the collecting device, and transport by a pipeline to the storage chamber, while airflow discharge from the other end of reservoir flower chamber, realize the separation of the gas and petals, and ultimately complete the safflower harvesting, transporting and collecting.

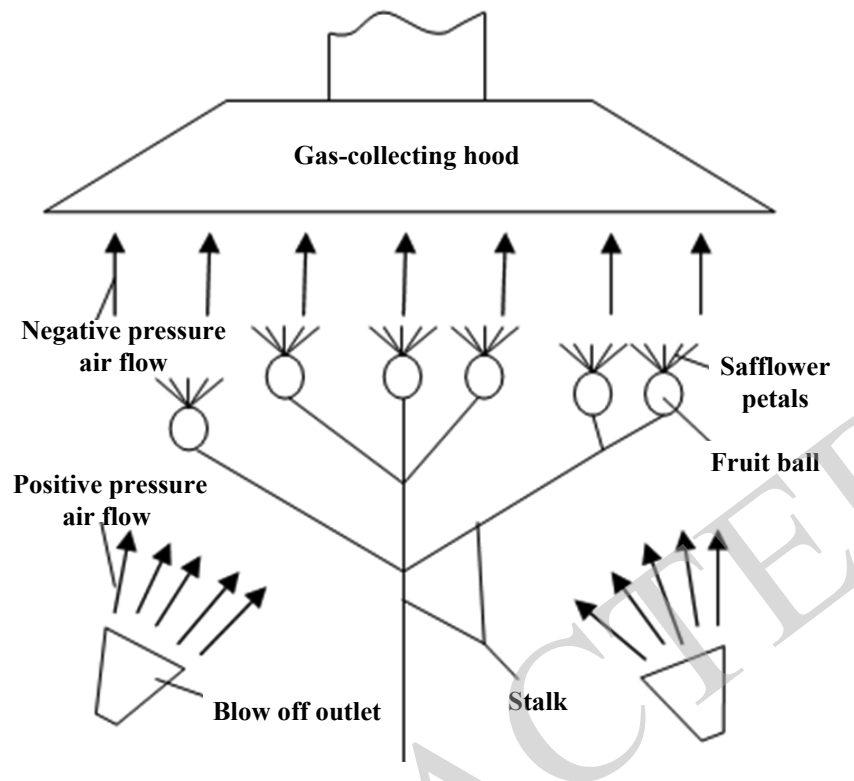

Fig. (1). Princle of safflowers' harvester.

\section{THE MODEL OF THE PNEUMATIC COLLECTED DEVICE}

Pneumatic collected device in the internal flow field the hood is decided to collect safflower petals direct factor of the transportation efficiency and effect. Reasonable design of the hood, and the harvesting apparatus in the use of under less wind can effectively collect and reduce the sloughing amount of stem filaments, on the other hand, even the wind is large not always reached better collected effect. In order to accurate simulation of flow field distribution, the gas hood number and a gas outlet set internal the cover shape parameter is the main factors.

\subsection{To Establish the Model of Conditions}

In the cover design should pay attention to the following points:

(1) In the cover design should pay attention to the following points: Plant should be set up surrounded by flower cover, the blowing backward diffusion limited petals in a minimum range, the basic structure and set size of flower cover should be based on macro parameters safflower plants (Fig. 2) as the basis;

In the cover design before and after the circular gap, safflower plant successfully can smooth internal the safflower set convenient cover;

In order to make full use of the original kinetic energy after being blown safflower petals, we should set the safflower suction mouth position and the flow direction safflower petals blown backward uniform;

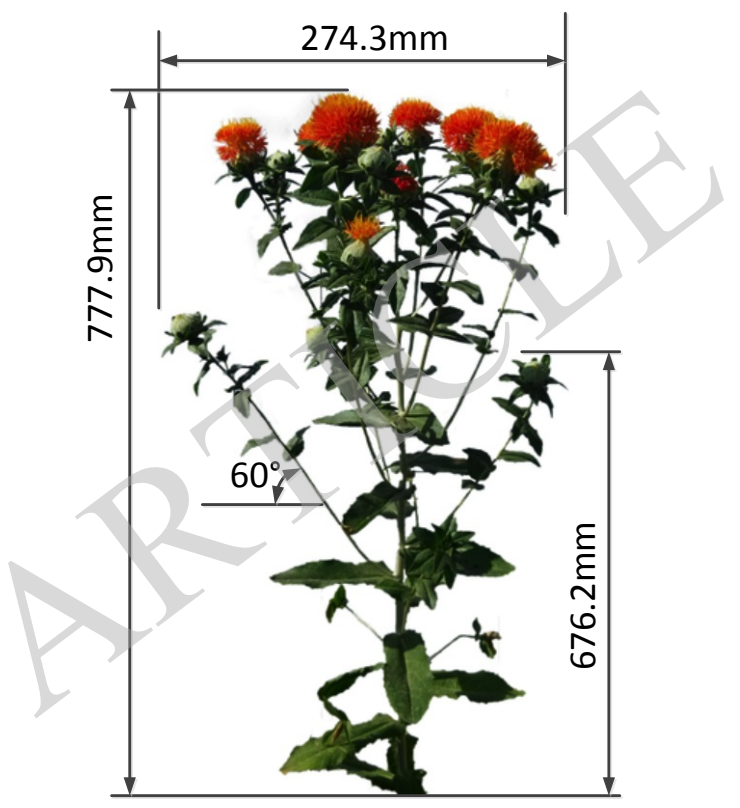

Fig. (2). The macroscopic schematics of safflower plants.

\subsection{Calculation of the Main Parameters Index Collected Device}

In the measure of the main technical index cover performance for exhaust air tate and pressure loss, the calculation method is given below the exhaust air tate and pressure loss :

\subsubsection{The Calculation of Exhaust Air Rate}

The theoretical analysis, in the hood exhaust aie rate:

$Q=V A-Q^{\prime}$

where : $V$ Through the cover of the velocity $/ m \cdot s^{-1} ; A$ - The total area of the hood $/ m^{2} ; Q^{\prime}$ — The air volume loss through gap. By the fimula 1, keep if the wind speed $V$ invariant, reduce the set safflower cover opening area $A$ exhaust air rate, $Q$ will be reduced correspondingly, thus make the system operation energy consumption to reduce.

\subsubsection{Pressure Loss Calculation}

the pressure loss of the safflowers hood;

$\Delta P=\xi P=\frac{1}{2} \xi \rho^{2} V^{2}$ 
where $\rho$ - flow density $/ \mathrm{Kg} \cdot \mathrm{m}^{-3} ; V$ - through the hood of the velocity $/ m \cdot s^{-1} ; \xi$ the pressure loss coefficient; and $\xi=\varphi^{-2}-1 ; \varphi$ the safflower hood flow coefficient, and $\varphi=\sqrt{\frac{P}{P^{\prime}}} ; P^{\prime}$ the static pressure in inspiratory steam tube; $P$-air flow pressure in the hood.

\subsection{Establishment of the Model}

In order to accurate simulation the internal flow field of the collecting device so as to improve the collection efficiency of the safflower petals; first of all, the design of closed type structure and no export and flow field simulation of the it; has the same structure respectively setting double and single outlet based on the analysis of the simulation results; the simulation, that a reasonable blow mouth position and the number of flowers, on this basis, to improve the external shape of the safflower hood. The walls were divided into parabolic and ladder cone type, its structure as shown in Fig. (3).
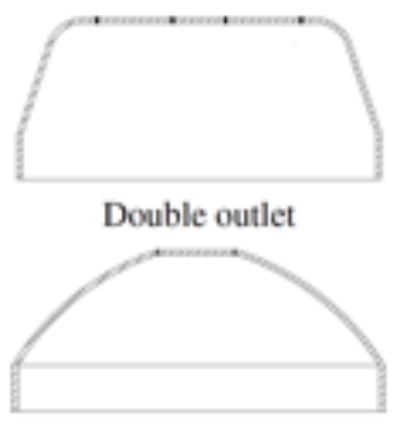

Parabola type
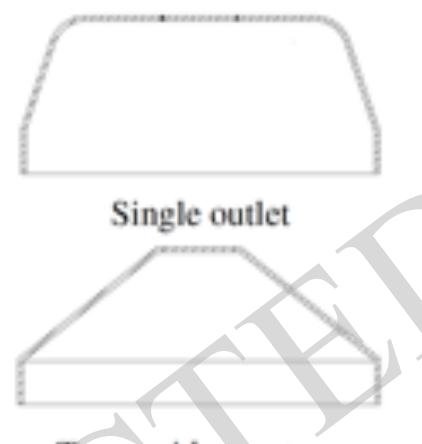

Trapezoid cone type
Fig. (3). The section graphs of different set flowers covers

\section{THE COLLECTION DEVICE'S AIRFLOW FIELD SIMULATION}

Results were observed and analyzed by choosing section I and section II (Fig. 4). To understand the flowing status of the airflow in the gas-collecting hood [13-15].

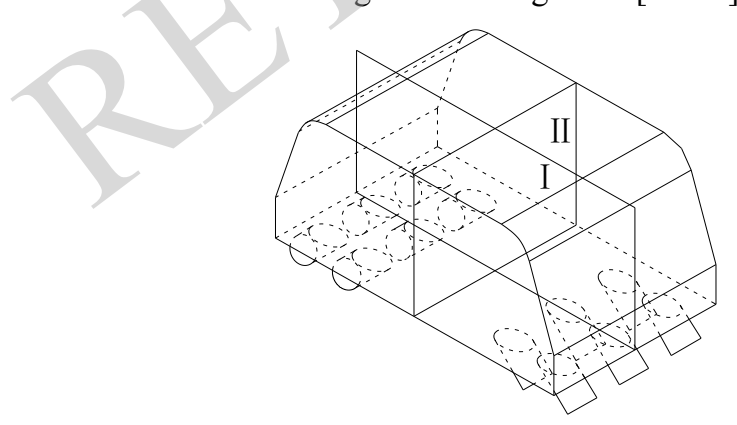

Fig. (4). The visualization section diagram of gas-collecting hood.

\section{The Simulation Results and Analysis of Flowers Cover Outlet's Number}

The velocity contour and vector picture of vector flowers cover as shown in Fig. (5). We can see these from the velocity contour and vector picture of sectional $\mathrm{I}$, there is a low-speed zone in the left and right sides and the upper region of the flowers cover, this is due to airflow of out of two sides of the inlet flow to deflect for two sides and upward after the collision occurred in the partial right side, downward flow along the sides of the wall after a collision with the upper part of wall, and forming a plurality of vortex region near each air inlet and the area between the upper wall and two shunt. We can see these from the velocity contour and vector picture of sectional II, the wind speed distribution compared with sectional I, two streams deflect for two sides of sectional II after the collision, thereby forming the low-speed area in the upper part of airflow after the collision, due to the velocity distribution, after the collision airflow flow from both sides of the wall to the top, collision again airflow flow down and forming a vortex. This closed type flow field is not conducive to collect the blown safflower, It collect inefficient and can easily lead safflower to fall.

\subsection{The Airflow Field Optimization of the Number of Gas-Collecting Hood}

According to the flow field flowing characteristics, we make the following changes for the structure of flowers cover : open double outlet at the top of the middle part in order to carry safflower airflow flowing when air is flowing.

We can see these from the velocity contour and vector picture (Fig. 6) of sectional I of double outlet structure, The airflow flow out from the both sides of air inlet, collision in the middle flowers cover and respectively flow to each side of the outlet, and the airflow on the right side converge with tributaries on the left lower side then flow toward the outlet, this will result in a small amount of wind on the left side of the outlet, It will make the safflower being picked in left part can't be collected due to lack of wind; and airflow in the right appear a larger deflection in direction during exercise, resulting in a large low-speed zone on the right side of the central, this will result in safflower planting on the right side can't be separated with cones because the airflow can't be reached or the wind is not enough. In a word, when setting double outlet, although it conform to internal air flowing regularity of closed type collection device, but collection device is easy to form a plurality of low-speed area in the internal, and airflow is uneven at the double outlet, it likely cause some filaments can't be collected.

Therefore, design a single outlet in the middle of the upper side on the collection device, we can see these from the velocity contour and vector picture of sectional I, both sides of the inlet airflow convergence in the lower part of the center, the area of the central high velocity air large, wind speed at the outlet is about $18 \mathrm{~m} / \mathrm{s}$, it's more than $14 \mathrm{~m} / \mathrm{s}$ of double outlet's wind speed. So choosing the single export structure collection device is conducive to collect safflower.

\subsection{The Simulation Results and Analysis of the Shape Parameters of Safflower Gas-Collecting Hood}

From the safflower collected cover of single export with the flow field on small taper shape simulation results we can see: its internal the right position appears phenomenon on the 

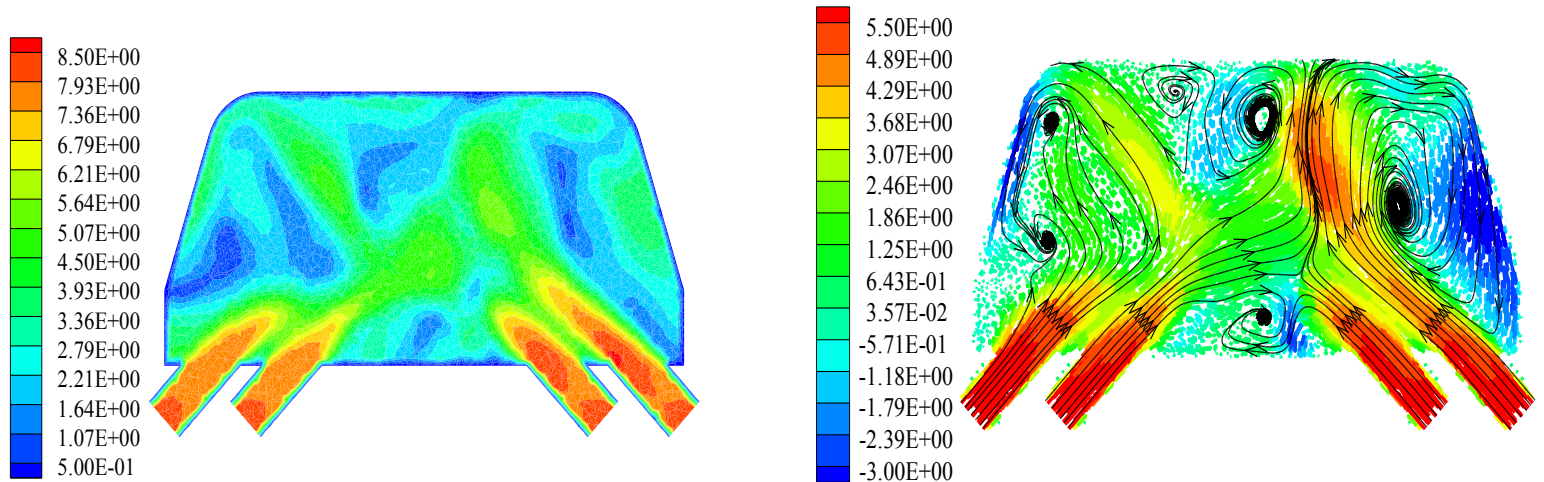

(a) Velocity Contour and velocity vector distributions of hermetic type gas-collecting hood at section I
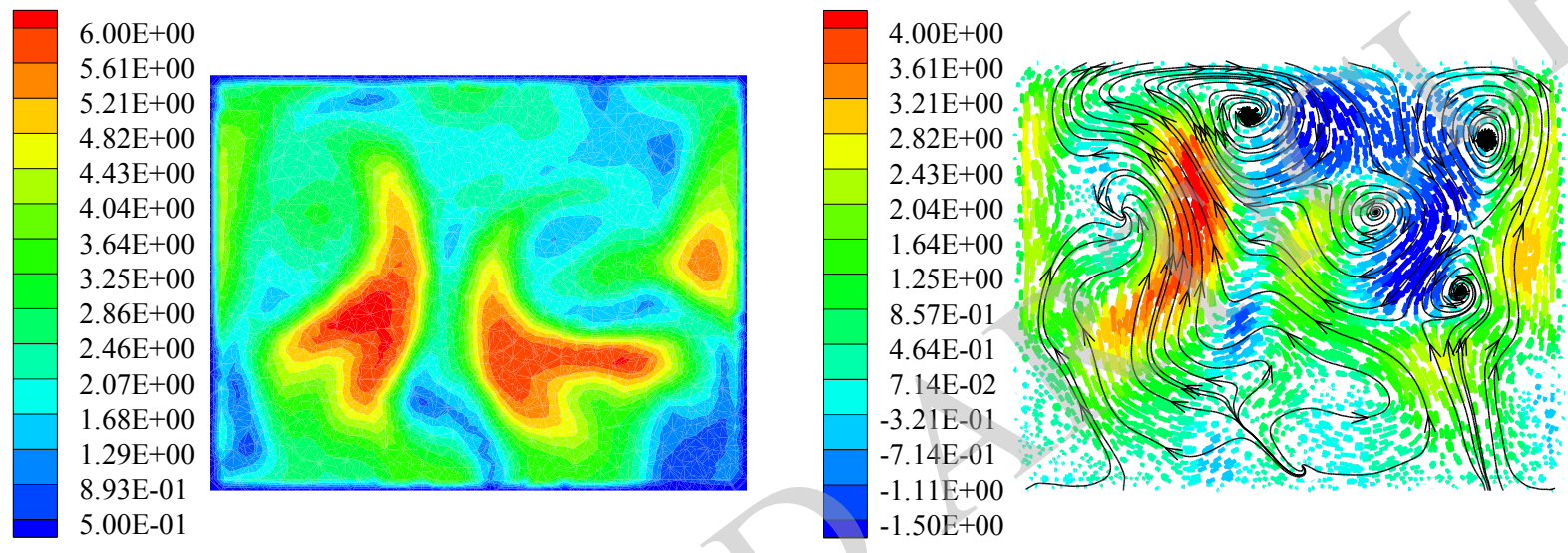

(b) Velocity Contour and velocity vector distributions of hermetic type gas-collecting hood at section II

Fig. (5). Flow-field simulation of set flowers cover without export.
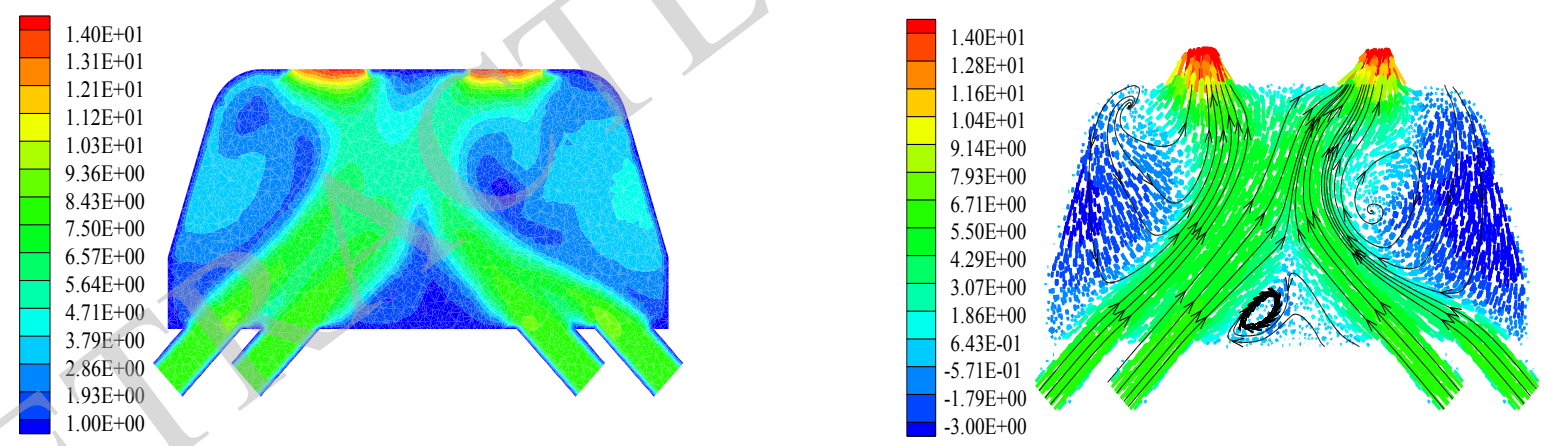

(a) Velocity Contour and velocity vector distributions of double outlet gas-collecting hood at section I
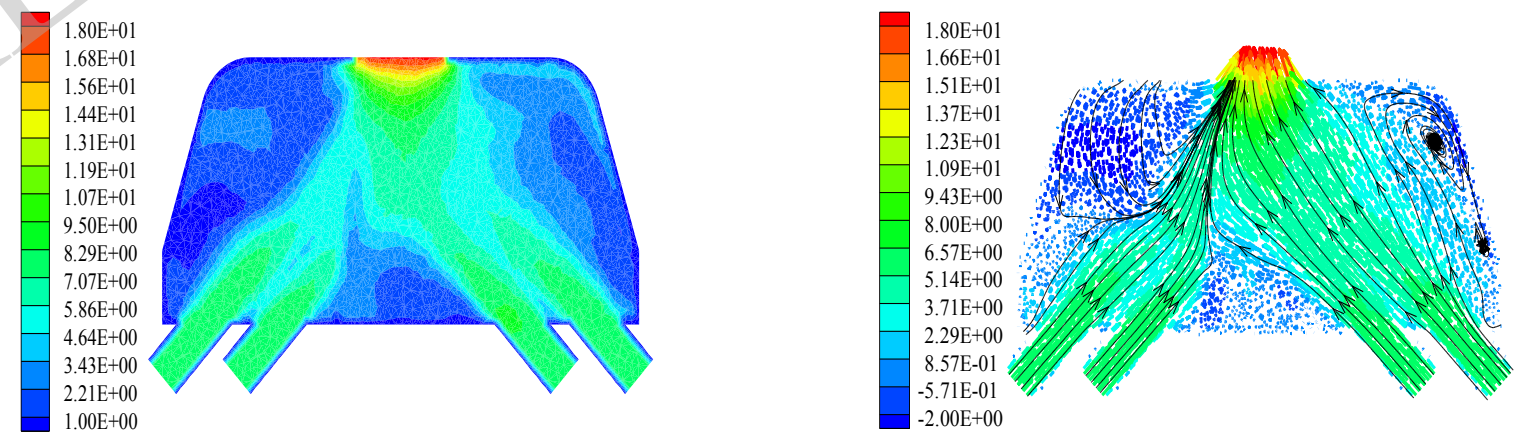

(b) Velocity Contour and velocity vector distributions of single outlet gas-collecting hood at section I

Fig. (6). Flow-field simulation of set flowers cover with different export number. 
flow shifting right, and finally forming vortex. According to the vortex theory, the local area of the vortex will generate induced velocity field weakening the wind speed at the outlet for the entire flow space, this will cause the loss of part of the air volume and the energy. From the velocity vector can also be seen flow of cross-sectional I appeares phenomenon that flow diffuses from bilateral of the wall to outlet flowing, this will make some wind not converge to the middle region, and do full of flow in the absence of the safflower area, and cause a waste of energy. Therefore, we improve the collection device structure based on the external shape to get better effect of airflow convergence on the basis of this structure.

The cross-sectional I velocity contour picture and velocity vector diagram of the collection device structure with parabolic (Fig. 7a) shows confluence of bilateral inlet flow is better and well-distributed of wind speed of safflower of bilateral contacting area being about $10.7 \mathrm{~m} / \mathrm{s}$ in internal safflower collected cover. Wind speed of transporting safflower area is about $5.86 \mathrm{~m} / \mathrm{s}$ after the confluence of flow, reaching requirements of blowing fallen, collection and delivery safflower. Just confluence region area of flow is small, in which there is a larger part of the low velocity zone $(2.21 \mathrm{~m} / \mathrm{s})$. Flow can be seen mainly in the upper of safflower collected cover from speed contour (Fig. 7b) of the crosssectional II , and the wind speed is too low $(2.21 \mathrm{~m} / \mathrm{s})$ below the central region. This will affect harvest safflower in the lower and partial bilateral middle region, reducing harvesting yield of safflower ultimately. The velocity vector diagram of cross-sectional II also shows three blowing-ports don't gather in cross-sectional II, forming three initial positions of wind separately, this will form a gap between each blowing- port, whose wind speed is not small enough to blow safflower in the region.

\subsection{The Optimization of Flow Field on the Shape Parameter of Safflower Collected Cover}

We increase the high-speed region of the central portion, and reduce low velocity zone of convergence of bilateral flow to make wind condensation furtherly. The parabolic structure of collecting device is changed into trapezoid cone type. The angle on inner row air inlet of bilateral air inlet pipeline is adjusted 40 degrees. The designing ideas are based on separation device adjusting the air inlet angle of bilateral each row to intermediate 30 degrees. The simulation results are shown in Fig. (8).

Compared with simulation results of parabolic collecting device, velocity contour picture and velocity vector diagram (Fig. 8a) of cross-sectional I with trapezoid cone type shows high speed area of the middle part in collecting device is increased, and low speed area in the lower part has been reduced after the confluence. Bilateral flow flows at the outlet smoothly, making filaments on the cross-sectional direction good picked, collected and transported.

The velocity contour picture and velocity vector diagram (Fig. 8b) of the cross-sectional II shows apparently three flow ahead under the premise of not reducing the wind speed have converged into a share, greatly reducing the low velocity zone between the separating device.

The result of flow field simulation with comprehensive analysis of the structure of each collecting device shows the simulation of flow field on collecting device internal

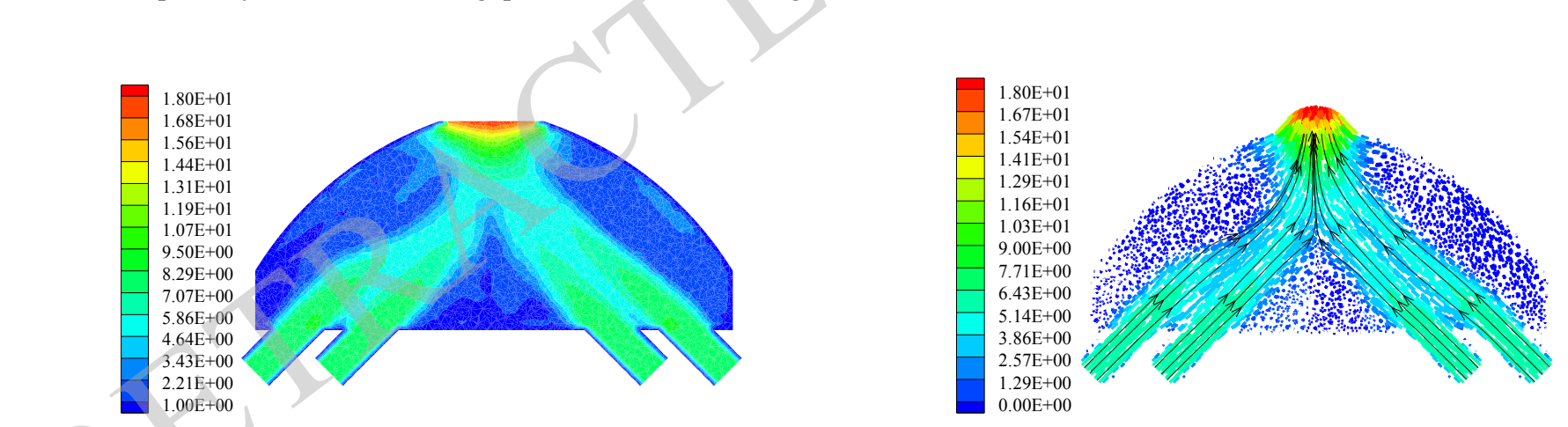

(a) Velocity Contour and vector distributions at section I
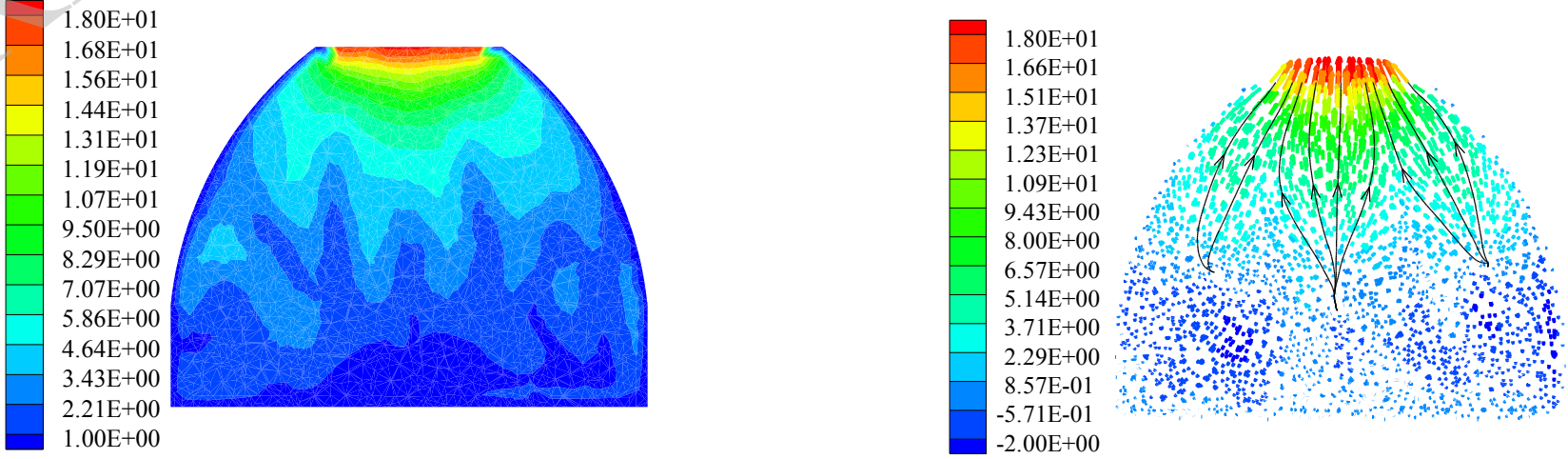

(b) Velocity Contour and vector distributions at section II

Fig. (7). Flow-field simulation of gas-collecting hood structure with parabolic. 

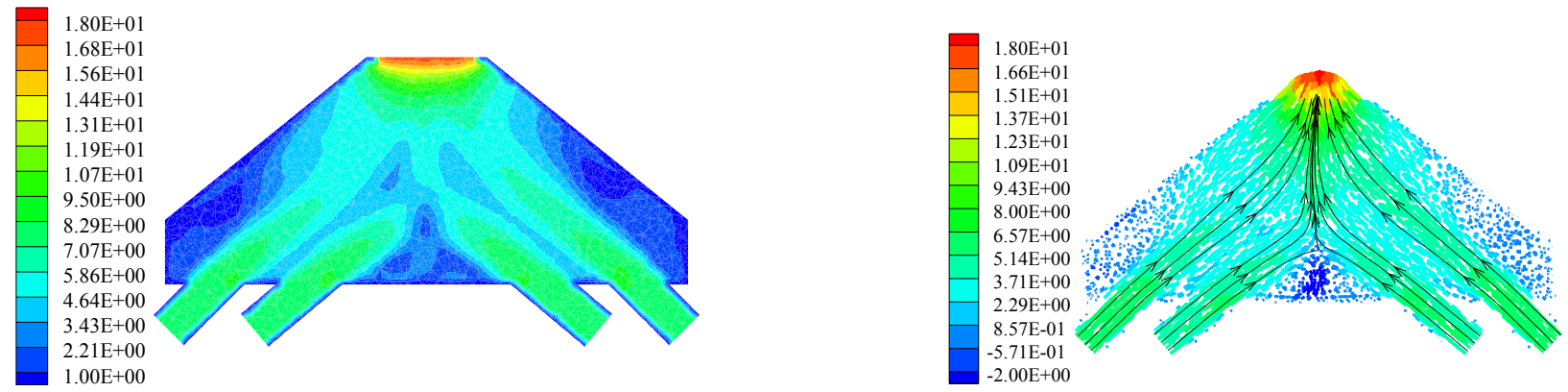

(a) Velocity Contour and vector distributions at section I
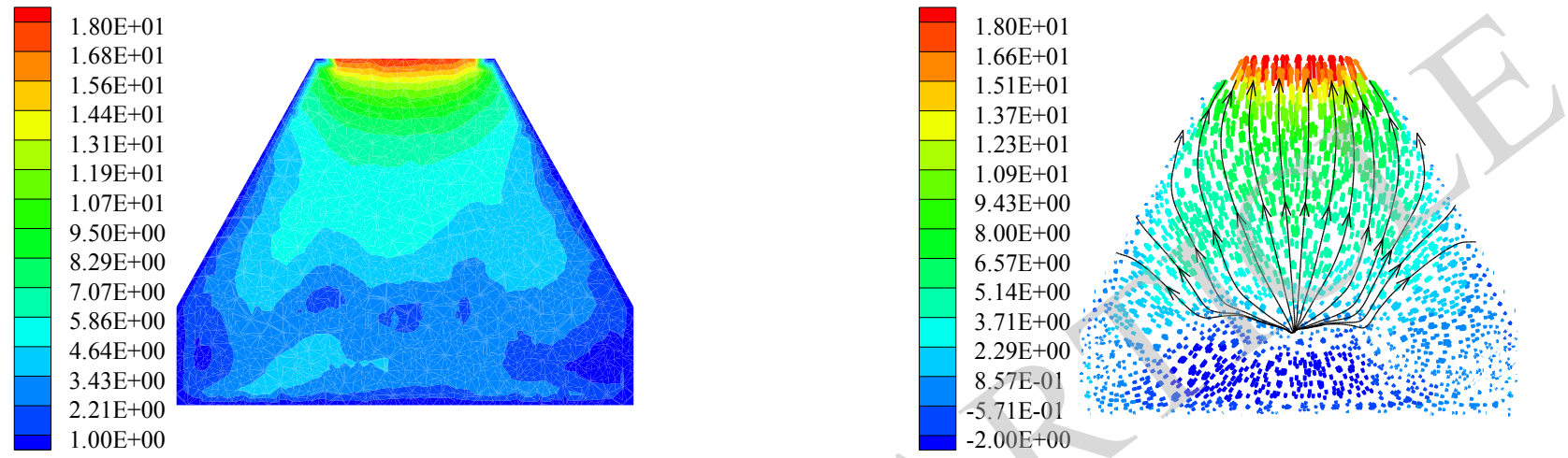

(b) Velocity Contour and vector distributions at section II

Fig. (8). Flow-field simulation of gas-collecting hood structure with trapezoid cone.

structure of inclined trapezoidal cone type with bilateral blowing flower-like-shape mouth is the best, being largearea of high-speed and no low-free zone: it being energy saving and easy of manufacturing can meet the requirements of filaments on picking, collecting and transportation.

\section{RESULTS}

(1) According to fluid simulation of collecting device structure for different number of exports. The distribution of flow field with design of single outlet at the center of collecting device is more suited to collect safflower by analysis.

(2) The distribution of flow field of safflower collected cover with trapezoid cone type is better than that of parabolic type structure.

(3) The result of numerical simulation of internal structure with trapezoidal cone-type by FLUENT software shows the area of internal high-speed flow is larger, and the original three streams of longitudinal section are converged into one share to reduce the gap between the pipe blowing flowers, form no low velocity zone, save energy, and manufacture easily.

\section{CONFLICT OF INTEREST}

The authors confirm that this article content has no conflict of interest.

\section{ACKNOWLEDGEMENTS}

This work is supported by the national natural sciencefoundation of china (No.51365048); the High-tech Research Project of Xingjiang, China (No. 201511107)

\section{REFERENCES}

[1] P.E. McGuire, A.B. Damania, and C.O. Qualset, "Safflower in California. The Paulden F. Knowles personal history of plant exploration and research on evolution, genetics, and breeding," Agronomy Progress Report No. 313, Dept. of Plant Sciences, University of California. Davis CA USA. 2012.

[2] Z. Wang, and Y. Chen, "Safflower" Beijing: Chinese Medicine Press, pp. 26-39, 2001.

[3] Y. Ge, L. Zhang, D. Han, J. Chen, and W. Fu, "Current state and development trend of the mechanical harvesting on saffron filaments," Journal of Agricultural Mechanization Research, vol. 36, no.11, pp.265-268, 2014. (in Chinese with English abstract)

[4] A. K. Rajvanshi, "Development of Safflower Petal Collector," Nimbkar Agricultural Research Institute (NARI), pp.1 6, 2005.

[5] S. Azimi, G. Chegini, M. H. Kianmehr, and A. Heidari, "Design and construction of a harvesting safflower petals machine," Mechanics and Industry, vol. 13, no.5, pp.301-305, 2012.

[6] D. Karayel, "Performance of a modified precision vacuum seeder for no-till sowing of maize and soybean," Soil and Tillage Research, vol.104, no.1, pp. 121-125, 2009.

[7] M.E.N.G. Zhipeng, W.A.N.G. Shulan, and D.I.N.G. Xinwei, "Three dimensional numerical simulation of flammable gas dispersion around an obstacle," Chemical Equipment Technology, vol. 28, no.2, pp. 74-77, 2007.

[8] Q. Wei, Y. Shi, W. Dong, et al. "Study on hydraulic performance of drip emitters by computational fluid dynamics," Agricultural Water Management, vol. 84, no.1/2, pp. 130-136, 2006. 
[9] P. Guarella, A. Pellerano, and S. Pascuzzi, "Experimental and theoretical performance of a vacuum seeder nozzle for vegetable seeds," Journal of Agricultural Engineering Research, vol. 64, no.1, pp. 29-36, 1996.

[10] M. Ilić, M. Petkovska, and A. Seidel-Morgenstern, "Determination of competitive adsorption isotherms applying the nonlinear frequency response method," Journal of Chromatography A, vol. 1216, no. 33, pp.6098-6107, 2009.

[11] Q. Feng, X. Wang, W. Zheng, Q. Qiu, and K. Jiang, "A new strawberry harvesting robot for elevated-trough culture," Int $J$ Agric \& Biol Eng, vol.5, no.2, pp.1-8, 2012.

[12] P. Tarrío, A. M. Bernardos, J. R. Casar, and J. A. Besada, "A harvesting robot for small fruit in bunches based on 3-D stereoscopic vision. Computers in Agriculture and Nature
Resources," In: ASABE 4th World Congress Conference, Publication Number: 701P0606, 2006.

[13] Y.P. Banjare, R.K. Sahoo, and S.K. Sarangi, "CFD simulation and experimental validation of a GM type double inlet pulse tube refrigerator,"

[14] V. M. Molochnikov, N. I. Mikheev, and O. A. Dushina, "Simulation of subsonic flows with separation using the FLUENT program package: software applicability study," Thermophysics and Aeromechanics, vol.16, no.3, pp.367-373, 2009.

[15] R. Sui, J. Alex, Thomasson, S. D. F. To, "Cotton-harvester-flow simulator for testing cotton yield monitors," Int J Agric \& Biol Eng, vol,3, no.1, pp.44-49, 2010.

(C) Yun et al.; Licensee Bentham Open.

This is an open access article licensed under the terms of the (https://creativecommons.org/licenses/by/4.0/legalcode ), which permits unrestricted, non-commercial use, distribution and reproduction in any medium, provided the work is properly cited. 